\title{
Vessel co-option in glioblastoma: emerging insights and opportunities
}

\author{
Giorgio Seano ${ }^{1} \cdot$ Rakesh K. Jain ${ }^{2}$
}

Received: 12 September 2019 / Accepted: 5 October 2019 / Published online: 2 November 2019

(c) The Author(s) 2019

\begin{abstract}
Vessel co-option is the movement of cancer cells towards and along the pre-existing vasculature and is an alternative to angiogenesis to gain access to nutrients. Vessel co-option has been shown as a strategy employed by some glioblastoma (GBM) cells to invade further into the brain, leading to one of the greatest challenges in treating GBM. In GBM, vessel cooption may be an intrinsic feature or an acquired mechanism of resistance to anti-angiogenic treatment. Here, we describe the histological features and the dynamics visualized through intravital microscopy of vessel co-option in GBM, as well as the molecular players discovered until now. We also highlight key unanswered questions, as answering these is critical to improve understanding of GBM progression and for developing more effective approaches for GBM treatment.
\end{abstract}

Keywords Vessel co-option $\cdot$ Glioblastoma $\cdot$ Anti-angiogenesis $\cdot$ Cell migration $\cdot$ Tumor microenvironment

\section{Introduction}

Tumors depend on blood vessels for growth and dissemination, making the tumor vasculature a compelling therapeutic target to limit tumor growth and metastasis. Angiogenesis, which is the formation of new vessels from pre-existing ones, is the most well-studied mechanism for tumors to generate vasculature. However, some tumors grow in already highly vascularized organs like liver, lung, lymph nodes, and brain, reducing the need to induce angiogenesis. As a consequence, tumor cells that are infiltrative have easy access to well-perfused pre-existing blood vessels. Vessel co-option, which we define here as the movement of tumor cells towards and then along the pre-existing blood vessels, is one mechanism to access the vasculature.

A compelling example of an infiltrating tumor is glioma-a highly vascularized tumor. Gliomas are the most

Giorgio Seano

giorgio.seano@curie.fr

Rakesh K. Jain

jain@steele.mgh.harvard.edu

1 Tumor Microenvironment Laboratory, Institut Curie Research Center, Paris Saclay University, PSL Research University, Inserm U1021, CNRS UMR3347, Orsay 91405, France

2 Edwin L. Steele Laboratory, Department of Radiation Oncology, Massachusetts General Hospital and Harvard Medical School, Boston, MA 02114, USA common malignant primary tumors growing in the central nervous system in adults, constituting approximately $80 \%$ of the malignant cases [1]. Gliomas can be divided into three sub-classes: oligodendrogliomas, astrocytomas, and glioblastomas (GBMs). GBM is one of the most deadly types of cancer with a median overall survival of 15 months [2, 3]. Despite massive clinical and research efforts, GBM treatment remains one of the most challenging tasks in clinical oncology [4]. Since the brain tissue is highly vascularized, just 3-6 glioma cells are needed to fill the space between two adjacent microvessels [5]. This estimation stresses the ease with which GBM cells could be in contact with already well-perfused blood vessels without the need to activate the pathways of tumor angiogenesis. Vessel co-opting GBM cells benefit from both their oxygen and nutrient supply and the specific vascular niche microenvironment that stimulates proliferation and self-renewal mediated by crosstalk with the cellular components of blood vessels. Moreover, infiltrating GBM cells employing vessel co-option use the vasculature as a scaffold to invade into normal CNS tissue.

There are a number of preclinical studies showing that several other types of tumor cells may migrate along the normal pre-existing vasculature [6-8], such as non-smallcell lung cancer [9] and uveal melanoma [10,11], as well as metastases from melanoma, breast, and colorectal cancer in the lymph node [12, 13], liver [14-16], or brain [15, 17-19]. Thus, vessel co-option may be a widespread and general strategy of growth for infiltrating tumors. 


\section{Vessel co-option as one of the GBM spreading strategies}

Almost all GBM patients experience tumor recurrence, typically occurring within 1-2 cm of the original tumor border [20]. Indeed, non-proliferating, infiltrating GBM cells [21] move very fast in the surrounding tissue with a velocity of 2 to $6 \mu \mathrm{m} / \mathrm{h}$ in preclinical models [22]. Their migration is characterized by a diffuse, fast, and undirected movement or by a slower, invasive, directional migration [22]. Histologically, GBM cells infiltrate the surrounding tissue through four different invasion pathways: (i) individual-cell migration within the extracellular matrix (diffuse infiltration), (ii) collective invasion of the surrounding tissue, (iii) peri-neuronal satellitosis, and (iv) perivascular migration (also called as vessel co-option) [8, 22-24] (Fig. 1). Specifically, vessel co-option has been hypothesized to be a significant contributor to recurrence and lethality as it enables the formation of microscopic tumor extensions beyond the margins of surgical resection $[25,26]$. Tumor infiltration is probably one of the most clinically relevant biological consequences of vessel co-option in GBMs. Indeed, vessel co-option has been shown as a preferred invasion strategy for some GBM cell lines [5]. However, the studies performed until now are limited as they comprise few patient-derived or syngeneic cell lines [e.g., C6 (rat), GL261 (mouse), and D54 and MGG8 (patient derived)], and thus, are unable to reflect the high complexity of GBM where different subtypes are present

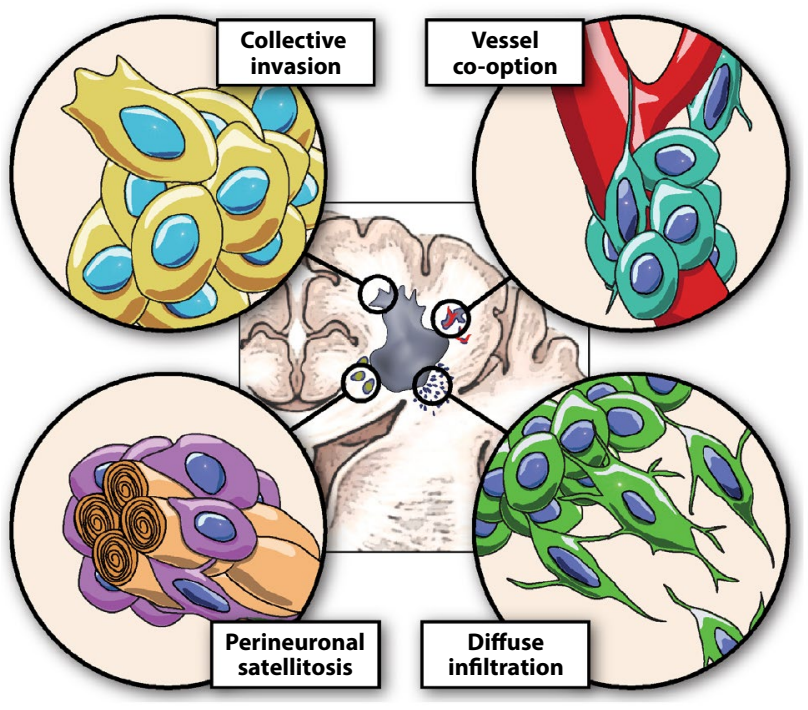

Fig. 1 Strategies used by GBM for infiltration into the surrounding brain tissue. Collective invasion of the surrounding tissue, perivascular migration (also called as vessel co-option), peri-neuronal satellitosis, and individual-cell migration within the extracellular matrix (diffuse infiltration) with an evident inter- and intra-tumor heterogeneity [27]. It is thus conceivable that different subtypes could employ distinct invasion strategies resulting in a more complex scenario where in the same tumor we encounter areas, subpopulations, or individual cells that differentially prefer vessel co-option, peri-neuronal satellitosis, individual infiltration, or collective invasion.

\section{Insights from in vivo imaging of vessel co-option}

Initially, the existence of vessel co-option was deduced from examination of histological tissue sections [28, 29]. Indeed, (i) the frequent vascular association of infiltrating tumor cells, as occurring in perivascular satellitosis, (ii) the presence of normal blood vessels and (iii) the maintenance of the normal vascular architecture in the infiltrated areas are signs of bona fide vessel co-option in GBMs; but they do not reveal the dynamics of the movement of tumor cells towards pre-existing vasculature. In the last decade, improvements in imaging technologies have made it possible to visualize vessel co-option in organotypic cultures and in live animals.

\section{Organotypic brain slices}

In order to observe the ex vivo dynamics, GBM vessel co-option has been recapitulated using organotypic brain slices cultured with patient-derived GBM cells [30]. Interestingly, GBM cells get in contact with blood vessels in this setup and this model shows interesting features of vessel co-option. First, GBM cells are "attracted" by blood vessels even without a gradient of nutrients and oxygen, since blood vessels are not perfused in brain slices. This suggests that there are chemoattractants produced and released by blood vessels, as discussed below. Secondly, the interaction between tumor cells with components of blood vessels is very dynamic. Moreover, one study revealed that GBM cells employ Cdc42-dependent and actin-based cytoplasmic extensions to modify the normal activity of pericytes around capillaries [30].

\section{Imaging of serially excised tissue}

Another interesting report sheds light on the in vivo occurrence of vessel co-option. The authors quantified the GBM cells' association with blood vessels by analyzing the histology of multiple animals sacrificed at different intervals from tumor implantation [5]. The syngeneic GL261 GBM model at the early stage of tumor progression showed massive perivascular invasion in the infiltrative area [5].

An additional study analyzed multiple patient-derived cell lines implanted in nude mice and demonstrated that 
GBM cells at the infiltrative area are associated more with capillaries (less than 7- $\mu \mathrm{m}$-diameters) than venules/arterioles [31]. Moreover, ultra-structural micrographs of GBM cells associated with blood vessels showed clear cell-to-cell contacts of GBM cells with endothelial cells [31].

\section{In vivo imaging}

The intravital microscopy can provide powerful insights into the dynamics of vessel co-option. Two studies using intravital microscopy - one from our laboratory and the other from Dr. Frank Winkler's Laboratory_-showed that GBM cells preferentially use blood vessels in the GL261 mouse glioma model to spread in a directional manner [32, 33]. Vessel co-opting GBM cells were frequently found next to multiple capillary structures where microvessels are parallel [32] and were strongly increased by anti-angiogenesis treatment [33]. Our second intravital time-lapse imaging study of patient-derived GBM cells implanted orthotopically showed that GBM cells closely interact with blood vessels, and move towards and then along the pre-existing brain vasculature [25] (Fig. 2). Vessel co-option appears to occur predominantly at the tumor-brain interface and in some GBM subtypes it is a fairly frequent phenomenon occurring in more than $50 \%$ of cells. Intravitally, as previously shown on brain slices, GBM cells form close and dynamic contacts with GFP-tagged endothelium [25].

\section{Vessel co-option molecular pathways}

Although the existence of vessel co-option has been hypothesized for some time [28, 29], the molecular pathways involved in the process of GBM vessel co-option are beginning to emerge now (Table 1).

\section{Bradykinin}

Bradykinin, a member of the kinins, is an endothelial cellcleaved product of high molecular weight kininogen [34]. Bradykinin is physiologically present in the brain and is increased during tumor progression. Moreover, vessel coopting GBM cells expresses high levels of bradykinin receptor-2 (B2R) [34]. Bradykinin induces chemotaxis in GBM cells and increases the GBM invasion of the surrounding brain tissue [35]. Interestingly, pharmacological or genetic inhibition of B2R in GBM cells impairs vessel co-option [34]. Of note, the bradykinin receptor inhibitor Icatibant, already FDA-approved for the treatment of acute attacks of hereditary angioedema, may be an interesting option for novel anti-vessel co-option treatment in GBM [23].

\section{CXCR4/SDF-1a}

Stromal cell-derived factor (SDF)-1 $\alpha$, also known as CXCL12, is a member of the CXC subfamily of chemokines and interacts with the seven-transmembrane G-proteincoupled receptor CXCR4. Originally, chemokines and their receptors were shown to be potent regulators of chemotaxis and trans-endothelial migration in leukocytes. They have also been described as potential chemotactic cues in tumors [36]. SDF1 $\alpha$ has been shown to be expressed in neurons, blood vessels, and white matter tracks, and all components
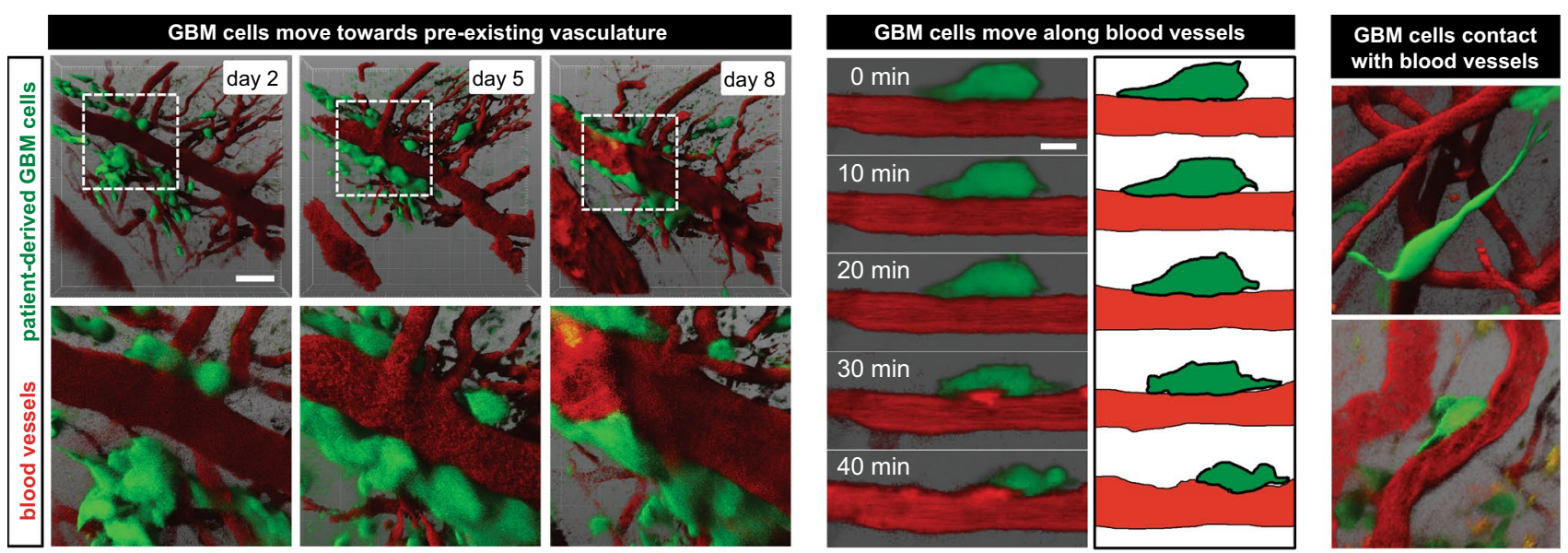

Fig. 2 Intravital microscopy of GBM vessel co-option dynamics. Time-lapse imaging demonstrating that GBM cells move towards and then along the pre-existing blood vessels, maintaining very close contact. Reproduced and adapted from [25] 
Table 1 Vessel co-option pathways in GBMs

\begin{tabular}{|c|c|c|c|}
\hline Pathways & Experimental model & Notes & References \\
\hline Bradykinin & $\begin{array}{l}\text { Patient-derived D54 in vivo model and in vitro co- } \\
\text { culture }\end{array}$ & $\begin{array}{l}\text { Bradykinin is released from blood vessels, while GBM } \\
\text { cells express B2R, their inhibition impairs vessel } \\
\text { co-option }\end{array}$ & {$[34,35]$} \\
\hline CXCR4/SDF1 $\alpha$ & G1261 mouse in vivo model and in vitro co-culture & $\begin{array}{l}\text { SDF1 } \alpha \text { is expressed in neuronal and endothelial cells, } \\
\text { while GBM cells express CXCR4, their inhibition } \\
\text { impairs vessel co-option and radiosensitizes tumors }\end{array}$ & {$[33,37-39]$} \\
\hline Ang-2 & C6 rat in vivo model & $\begin{array}{l}\text { Ang-2 and VEGF are expressed in vessel co-option } \\
\text { areas as a consequence of vascular regression }\end{array}$ & {$[33,43]$} \\
\hline IL-8 & In vitro co-culture and in vivo implants & $\begin{array}{l}\text { Endothelial cells increase GBM invasiveness and tumor } \\
\text { growth through IL-8-mediated enrichment of glioma } \\
\text { stem cells }\end{array}$ & {$[46,47]$} \\
\hline EGFRvIII & Mouse in vivo model and ex vivo brain slice & $\begin{array}{l}\text { EGFRvIII-hi GBM cells are highly vessel co-opting } \\
\text { and tumors originated by them are highly infiltrative } \\
\text { and aggressive }\end{array}$ & [49] \\
\hline MDGI/FABP3 & Mouse in vivo model and ex vivo brain slice & $\begin{array}{l}\text { Modulation of MDGI/FABP3 strongly alters the GBM } \\
\text { cells' ability of infiltrating the surrounding brain tis- } \\
\text { sue with perivascular migration }\end{array}$ & {$[51,52]$} \\
\hline IRE- $1 \alpha$ & Neurospheres and U87 in vivo models & $\begin{array}{l}\text { Inhibition of IRE- } 1 \alpha \text { increases vessel co-option and } \\
\text { decreases pro-angiogenic pathways }\end{array}$ & {$[54,55]$} \\
\hline $\mathrm{CDC} 42$ & Ex vivo brain slice & $\begin{array}{l}\text { Vessel co-opting GBM cells co-opt and interact with } \\
\text { pericytes in a CDC42-dependent manner }\end{array}$ & {$[30]$} \\
\hline EphrinB2 & $\begin{array}{l}\text { Patient-derived MGG8 in vivo model, syngeneic model } \\
\text { and in vitro co-culture }\end{array}$ & $\begin{array}{l}\text { Endothelial Ephrin-B2 regulates vessel co-option, } \\
\text { when, and only if, the ligand Ephrin-B2 is upregu- } \\
\text { lated in GBM cells }\end{array}$ & {$[58]$} \\
\hline Olig2/Wnt7a & $\begin{array}{l}\text { Patient-derived MGG8 in vivo model, EGFRvIII- } \\
\text { induced syngeneic model and ex vivo brain slice }\end{array}$ & $\begin{array}{l}\text { Olig2-Wnt7 axis drives individual vessel co-option } \\
\text { in oligodendrocyte-like GBM cells, its inhibition } \\
\text { impairs vessel co-option and chemosensitizes tumors }\end{array}$ & {$[18,25]$} \\
\hline
\end{tabular}

CXCR4 CXC receptor-4, SDF1 $\alpha$ stromal cell-derived factor-1 $\alpha, I L-8$ interleukin 8, Ang-2 angiopoietin 2, CDC42 cell division control protein 42, EGFRvIII epidermal growth factor receptor variant III, MDGI/FABP3 mammary-derived growth inhibitor (MDGI)/fatty acid binding protein 3, IRE1 $\alpha$ inositol-requiring enzyme (IRE)-1 $\alpha$, Wht is acronym of homologous wingless (wg) and Int-1, Olig2 oligodendrocyte transcription factor

of the GBM secondary events of satellitosis. Moreover, stimulation with vascular endothelial growth factor (VEGF), typically present at the tumor-brain interface, upregulates SDF1 $\alpha$ in neurons and endothelial cells, while CXCR4 was found to be overexpressed in invading GBM cells [37]. In vitro, CXCR4+ GBM cells migrate towards a gradient of SDF1 $\alpha$ and inhibition of CXCR4 reduces GBM migration. Genetic or pharmacological inhibition of CXCR4 reduces invasion and improves survival in GBM as well as radiosensitizes tumors as measured by mouse survival and cell apoptosis [38]. Importantly, the SDF $1 \alpha / C X C R 4$ pathway was found to be upregulated by anti-angiogenic treatment [39], suggesting reciprocity between angiogenesis and vessel co-option (see below).

\section{Angiopoietin-2}

Angiopoietin-2 (Ang-2) and VEGF are the most important pro-angiogenic factors, produced and released by many tumors as a consequence of hypoxia in order to stimulate the formation of new blood vessels [40-42]. Although apparently counterintuitive, these mainly pro-angiogenic pathways have been shown to be present in vessel co-option areas at early stages of GBM formation. Indeed, Ang-2 is highly expressed in co-opted blood vessels in the C6 rat glioma model [43]. This report also described that after co-option multiple blood vessels regress with a consequent avascular tumor stage. During this vascular regression, the co-opting GBM cells begin expressing high levels of VEGF [43] and this may be the consequence of a reduction of perfusion in the co-opted/regressing blood vessels, with resulting hypoxia. Using a mathematical model, we described the dynamics of vessel co-option and showed that the vessel regression is caused by compression of vessels by the growth of cancer cells around co-opted vessels [33]. Although multiple other studies reported that vessel co-option is independent of anti-VEGF treatment or even induced by it $[5,14,15$, $25,44]$, the precise temporal role of Ang-2 and VEGF in vessel co-option areas and the dynamics of vascular regression of co-opted blood vessels needs to be further investigated using intravital microscopy. 


\section{Interleukin-8}

Interleukin-8 (IL-8) is a pro-inflammatory chemokine important in the initiation of neutrophil chemotaxis and degranulation. The receptors for it are two cell-surface G-protein-coupled receptors (CXCR1 and CXCR2). IL-8 has been shown to be particularly important for tumor progression and upregulates stem cell marker expression in GBM and other cancers [45]. In two distinct reports, co-culture of patient-derived GBM and endothelial cells was used to discover the chemotactic pathways activated by endothelial cells to stimulate GBM cell invasion. The authors showed that endothelial IL-8 increased GSCs invasiveness and growth $[46,47]$. Moreover, another report demonstrated that the IL-8-CXCR1/2 axis induces GBM proliferation, invasion, and vascular mimicry [48].

\section{EGFRvIII}

EGFRvIII, a mutation isoform formed by the deletion of exons 2-7 of the epidermal growth factor receptor (EGFR), is a common alteration in GBM. Using brain slice and in vivo orthotopic models, GBM cells with high EGFRvIII expression have been shown to be highly vessel co-opting and the tumors originated from them are highly infiltrative and aggressive [49]. This report did not investigate the molecular mechanisms of action of EGFRvIII-mediated vessel co-option, but suggests potential involvement of migration pathways and reduction of ECM adhesion [49].

\section{MDGI/FABP3}

Mammary-derived growth inhibitor (MDGI), also called heart-type fatty acid binding protein (H-FABP/FABP3), enables the intracellular transport of fatty acids [50]. MDGI/ FABP3 was found overexpressed in aggressive mesenchymal GBM and the tumor vasculature, which correlated with poor patient survival [51, 52]. Notably, modulation of MDGI/FABP3 strongly altered the GBM cells' ability to co-opt blood vessels as evident in the histological analysis [52]. Pharmacological targeting of the MDGI/FABP3 pathway using the antihistamine Clemastine strongly inhibited perivascular migration and invasive growth [52].

\section{Inositol-requiring enzyme (IRE)-1a}

Inositol-requiring enzyme (IRE)- $1 \alpha$ is an endoplasmic reticulum transmembrane protein and a cellular stress sensor [53]. Selective inactivation of IRE- $1 \alpha$ RNAse in GBM cells using dominant-negative approaches induces vessel co-option in the U87 tumor model and increases in vitro migration [54].
Interestingly, inhibition of IRE1 $\alpha$ also decreases all pathways for angiogenesis [55], thus confirming the hypothesis of angiogenesis/co-option reciprocity (see below).

\section{CDC42}

CDC42 is a key molecular actor for directional migration and drives the formation of filopodia at the leading edge of cells [56]. Using brain slices co-cultured with GBM cells, a report showed a close CDC42-dependent interaction between GBM cells and pericytes in co-opted vessels [30]. The report further showed that targeting Cdc42 function impairs GBM vessel co-option [30]. Although an interesting finding, CDC42 inhibition modifies the migratory and contractive ability of the GBM cells, inhibiting all migratory/ infiltration strategies and not specifically vessel co-option.

\section{Ephrin-B2}

Ephrin-B2 is a member of the Eph/ephrin family, a fundamental cell-to-cell communication system with widespread roles in tissue development, maintenance, and disease [57]. Using intravital imaging in murine GBM models and patient-derived cell lines, endothelial ephrin-B2 has been reported as an important regulator of vessel co-option if the ligand ephrin-B2 is also upregulated in GBM cells [58]. Moreover, the overexpression of ephrin-B2 in GBM cells was capable of transforming immortalized neural stem cells and inducing anchorage-independent growth. Importantly, genetic downregulation of ephrin-B2 impaired in vivo vessel co-option and improved survival [58].

\section{Olig2/Wnt7a: individual-cell versus collective-cell vessel co-option}

Patient-derived GBM cells may co-opt blood vessels as individual cells or as a collective cluster of cells. We recently demonstrated that the Olig2-Wnt7a signaling axis clearly induces individual-cell vessel co-option, making the tumors more infiltrative [25]. These data suggest that astrocyte-like GBM cells (i.e., Olig2- and Wnt7-negative) co-opt blood vessels mainly as collective clusters of cells, while OPC-like cells (i.e., driven by Olig2 and Wnt7) spread in the surrounding tissue as individual cells in association with blood vessels [25]. Astrocyte-like GBMs, prevalently characterized by collective vessel co-option, show disruption of the astrocytevascular coupling and blood-brain barrier (BBB) breach, with consequent blood vessel leakage, abnormal vasculature (large lumen and tortuous architecture), and inflammation caused by vessel leakage [25, 31]. In contrast, more OPClike GBMs with individual-cell vessel co-option are characterized by a much more subtle infiltration of the surrounding tissue, with no inflammation or vascular leakage. Thus, 
OPC-like tumors-or the tumor regions characterized by a prevalence of OPC-like cells-are theoretically undetectable by clinical imaging and are less inflammatory [25, 31] (Fig. 3). Importantly, Wnt7 can be targeted with inhibitors of porcupine in the endoplasmic reticulum, by reducing the secretion of multiple Wnt ligands [59]. Ex vivo and in vivo treatment with porcupine inhibitors showed a progressive reduction of cells in contact with blood vessels during Wnt inhibition. Moreover, porcupine inhibition showed chemotherapy sensitization in terms of survival of mice. Notably, vessel co-option inhibition through porcupine inhibition induced a more angiogenic phenotype, thus underlining the reciprocity between the two alternative vascular strategies [25].

\section{Intrinsic versus acquired resistance to anti-angiogenesis}

Vessel co-option can be an intrinsic feature of specific GBM subtypes or an adaptive ability acquired as a consequence of angiogenesis inhibition, such as with bevacizumab, sunitinib, etc. [41]. Clinically, studies have shown that vessel co-option occurs in GBM patients in the peritumor regions, and is increased in patients following anti-angiogenic treatment $[41,44]$. Thus, vessel co-option has been proposed as

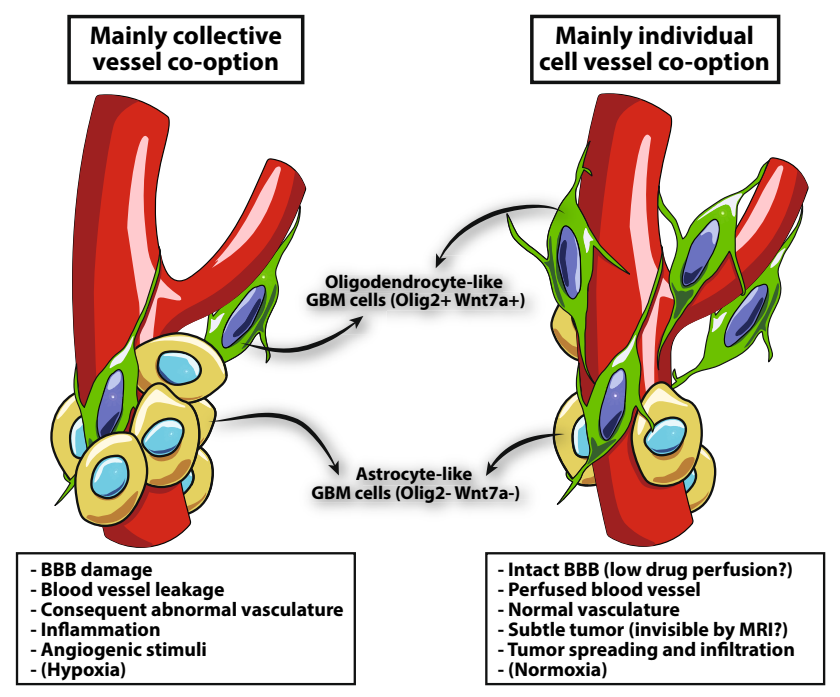

Fig. 3 Individual versus collective-cell vessel co-option. GBMs with a prevalent collective-cell vessel co-option are probably mainly composed of astrocyte-like GBM cells. These tumors show disruption astrocyte-vascular coupling and $\mathrm{BBB}$, with a consequent blood vessel leakage and abnormal vasculature (large lumen and tortuous architecture), inflammation caused by vessel leakage and an increase of angiogenic stimuli. While GBMs with more individual-cell vessel co-option are composed of a prevalence of OPC-like GBM cells. These tumors are characterized by a much more subtle infiltration of the surrounding tissue, with no inflammation and vascular leakage a resistance mechanism to anti-angiogenic therapy $[33,41$, 60, 61].

Patients with vessel co-opting tumors are intrinsically resistant to anti-angiogenic treatment, since they are not dependent on angiogenic factors, such as VEGF, Ang-2, or the fibroblast growth factor (FGF)-2 [5, 15, 26, 62]. Further, patients with co-opting tumors have the potential to reduce the overall efficacy of anti-angiogenic therapy measured in clinical trials even if many patients in the study cohort are responders with angiogenic tumors. Currently, there are no studies describing the rates of occurrence of angiogenesis or vessel co-option-driven GBMs in patients, which limits the ability to stratify patients in the trial design. This highlights the critical need of validated biomarkers capable of stratifying GBM patients regarding the extent of vessel co-option.

A second reason for the partial failure of anti-angiogenic treatments is certainly due to high GBM cell plasticity. As demonstrated in preclinical and clinical samples, anti-angiogenesis treatments induce vessel co-option in originally angiogenic tumors [25, 44]. Unfortunately, vessel co-option inhibition has also been shown to induce pro-angiogenic factors in treated cells and tumors [25]. These data show how GBMs can switch between vessel co-option and angiogenesis to meet their metabolic needs. Therapeutically, this suggests that inhibition of both pathways may reduce the occurrence of acquired resistance mechanisms [33]. Our recent computational model suggests that sequential inhibition of vessel co-option followed by anti-angiogenesis treatment could reduce GBM growth in comparison with the simultaneous blockade [33].

\section{Perspective}

The study of vessel co-option is an emerging field in vascular and tumor biology. It is likely that many types of tumors employ vessel co-option intrinsically or as a resistance mechanism to counter anti-angiogenic therapy. It is also conceivable that vessel co-option could influence multiple crucial features of tumors, such as hypoxia/normoxia, tumor metabolism, tumor invasion, immune suppression, and the presence of cells in specific resistance niches. While this mini-review has focused largely on vessel co-option and its relationship with angiogenesis, tumors can also recruit blood vessels by additional mechanisms: vasculogenesis, intussusception, and vascular mimicry [41, 60]. Relationships between these mechanisms and vessel co-option are virtually unknown. Our knowledge and classification of human tumors based on vessel co-option is still in its infancy and we lack histological and molecular signatures capable of stratifying GBM patients for the extent of vessel co-option. Finally, a better molecular understanding and well-characterized animal models will be needed to develop new 
therapeutic strategies aimed at inhibiting vessel co-option in preclinical models and in patients for combining with the approved and emerging treatments. This is even more critical as a result of the recent approvals of combinations of anti-angiogenic drugs and immune-checkpoint blockers for lung and kidney cancers $[63,64]$. These trials used antiVEGF agents that target primarily angiogenic blood vessels. However, the combination of bevacizumab (an anti-VEGF antibody) and an immune checkpoint blocking antibody has failed to improve survival of GBM patients in a randomized phase III trial. It remains unknown if and to what extent the inability of bevacizumab to target co-opted vessels in GBM played a role in this failure. Future studies need to address the role of vessel co-option in immunotherapy, a treatment that has revolutionized the therapy of more than 15 tumor types [63].

Acknowledgements We thank the Tumor Microenvironment Laboratory at Institut Curie as well as Drs. Timothy P. Padera, Shanmugarajan Krishnan, Nancy Wang, and Triantafyllos Stylianopoulos for critical reading and insightful suggestions. This work was supported by the Fondation ARC pour la recherche sur le cancer, the INSERM-CNRS ATIP-Avenir grant, the European Research Council (ERC) under the European Union's Horizon 2020 (Grant Agreement No. 805225), and the NanoTheRad grant from Paris-Saclay University (G.S.) as well as by US National Cancer Institute Grants P01CA080124, R01-CA129371, R01-CA208205, and U01-CA 224348; Outstanding Investigator Awards R35-CA197743; and Grants from the National Foundation for Cancer Research, Jane's Trust Foundation, Advanced Medical Research Foundation, and Harvard Ludwig Cancer Center (R.K.J.).

Open Access This article is distributed under the terms of the Creative Commons Attribution 4.0 International License (http://creativeco mmons.org/licenses/by/4.0/), which permits unrestricted use, distribution, and reproduction in any medium, provided you give appropriate credit to the original author(s) and the source, provide a link to the Creative Commons license, and indicate if changes were made.

\section{References}

1. Ostrom QT et al (2018) CBTRUS statistical report: primary brain and other central nervous system tumors diagnosed in the United States in 2011-2015. Neurooncology 20(Suppl_4):iv1-iv86

2. Koshy $\mathrm{M}$ et al (2012) Improved survival time trends for glioblastoma using the SEER 17 population-based registries. J Neurooncol 107(1):207-212

3. Gilbert MR et al (2014) A randomized trial of bevacizumab for newly diagnosed glioblastoma. N Engl J Med 370(8):699-708

4. Aldape $\mathrm{K}$ et al (2019) Challenges to curing primary brain tumours. Nat Rev Clin Oncol 16(8):509-520

5. Baker GJ et al (2014) Mechanisms of glioma formation: iterative perivascular glioma growth and invasion leads to tumor progression, VEGF-independent vascularization, and resistance to antiangiogenic therapy. Neoplasia 16(7):543-561

6. Donnem $\mathrm{T}$ et al (2013) Vessel co-option in primary human tumors and metastases: an obstacle to effective anti-angiogenic treatment? Cancer Med 2(4):427-436
7. Pezzella F, Gatter K (2015) Non-angiogenic tumours unveil a new chapter in cancer biology. J Pathol 235(3):381-383

8. Donnem T et al (2018) Non-angiogenic tumours and their influence on cancer biology. Nat Rev Cancer 18(5):323-336

9. Coelho AL et al (2016) Angiogenesis in NSCLC: is vessel cooption the trunk that sustains the branches? Oncotarget. https:// doi.org/10.18632/oncotarget.7794

10. Fornabaio G et al (2018) Angiotropism and extravascular migratory metastasis in cutaneous and uveal melanoma progression in a zebrafish model. Sci Rep 8(1):10448

11. Rodewald AK et al (2019) Eight autopsy cases of melanoma brain metastases showing angiotropism and pericytic mimicry. Implications for extravascular migratory metastasis. J Cutan Pathol. https://doi.org/10.1111/cup.13465

12. Jeong HS et al (2015) Investigation of the lack of angiogenesis in the formation of lymph node metastases. J Natl Cancer Inst. https://doi.org/10.1093/jnci/djv155

13. Pereira ER et al (2018) Lymph node metastases can invade local blood vessels, exit the node, and colonize distant organs in mice. Science 359(6382):1403-1407

14. Frentzas $\mathrm{S}$ et al (2016) Vessel co-option mediates resistance to anti-angiogenic therapy in liver metastases. Nat Med. https:// doi.org/10.1038/nm.4197

15. Leenders WP et al (2004) Antiangiogenic therapy of cerebral melanoma metastases results in sustained tumor progression via vessel co-option. Clin Cancer Res 10(18 Pt 1):6222-6230

16. Kuczynski EA et al (2016) Co-option of liver vessels and not sprouting angiogenesis drives acquired sorafenib resistance in hepatocellular carcinoma. J Natl Cancer Inst. https://doi. org/10.1093/jnci/djw030

17. Valiente $\mathrm{M}$ et al (2014) Serpins promote cancer cell survival and vascular co-option in brain metastasis. Cell 156(5):1002-1016

18. Kienast $\mathrm{Y}$ et al (2010) Real-time imaging reveals the single steps of brain metastasis formation. Nat Med 16(1):116-122

19. Er EE et al (2018) Pericyte-like spreading by disseminated cancer cells activates YAP and MRTF for metastatic colonization. Nat Cell Biol 20(8):966-978

20. Hou LC et al (2006) Recurrent glioblastoma multiforme: a review of natural history and management options. Neurosurg Focus 20(4):E5

21. Darmanis S et al (2017) Single-cell RNA-Seq analysis of infiltrating neoplastic cells at the migrating front of human glioblastoma. Cell Rep 21(5):1399-1410

22. Alieva $\mathrm{M}$ et al (2019) Intravital imaging of glioma border morphology reveals distinctive cellular dynamics and contribution to tumor cell invasion. Sci Rep 9(1):2054

23. Cuddapah VA et al (2014) A neurocentric perspective on glioma invasion. Nat Rev Neurosci 15(7):455-465

24. de Gooijer MC et al (2018) An experimenter's guide to glioblastoma invasion pathways. Trends Mol Med 24(9):763-780

25. Griveau A et al (2018) A glial signature and Wnt7 signaling regulate glioma-vascular interactions and tumor microenvironment. Cancer Cell 33(5):874-889.e7

26. Verhoeff JJ et al (2009) Concerns about anti-angiogenic treatment in patients with glioblastoma multiforme. BMC Cancer 9:444

27. Neftel C et al (2019) An integrative model of cellular states, plasticity, and genetics for glioblastoma. Cell 178(4):835-849.e21

28. Pezzella F, Gatter K, Qian CN (2016) Twenty years after: the beautiful hypothesis and the ugly facts. Chin J Cancer 35:22

29. Pezzella $\mathrm{F}$ et al (1996) Angiogenesis in primary lung cancer and lung secondaries. Eur J Cancer 32A(14):2494-2500

30. Caspani EM et al (2014) Glioblastoma: a pathogenic crosstalk between tumor cells and pericytes. PLoS ONE 9(7):e101402 
31. Watkins S et al (2014) Disruption of astrocyte-vascular coupling and the blood-brain barrier by invading glioma cells. Nat Commun 5:4196

32. Winkler $F$ et al (2009) Imaging glioma cell invasion in vivo reveals mechanisms of dissemination and peritumoral angiogenesis. Glia 57(12):1306-1315

33. Voutouri $\mathrm{C}$ et al (2019) Experimental and computational analyses reveal dynamics of tumor vessel cooption and optimal treatment strategies. Proc Natl Acad Sci USA 116(7):2662-2671

34. Montana V, Sontheimer H (2011) Bradykinin promotes the chemotactic invasion of primary brain tumors. J Neurosci 31(13):4858-4867

35. Seifert S, Sontheimer H (2014) Bradykinin enhances invasion of malignant glioma into the brain parenchyma by inducing cells to undergo amoeboid migration. J Physiol 592(22):5109-5127

36. Dewan MZ et al (2006) Stromal cell-derived factor-1 and CXCR36 receptor interaction in tumor growth and metastasis of breast cancer. Biomed Pharmacother 60(6):273-276

37. Zagzag D et al (2008) Hypoxia- and vascular endothelial growth factor-induced stromal cell-derived factor-1alpha/CXCR37 expression in glioblastomas: one plausible explanation of Scherer's structures. Am J Pathol 173(2):545-560

38. Yadav VN et al (2016) CXCR4 increases in-vivo glioma perivascular invasion, and reduces radiation induced apoptosis: a genetic knockdown study. Oncotarget. https://doi.org/10.18632/oncotarget .13295

39. Pham K et al (2015) VEGFR inhibitors upregulate CXCR39 in VEGF receptor-expressing glioblastoma in a TGFbetaR signalingdependent manner. Cancer Lett 360(1):60-67

40. Seano $\mathrm{G}$ et al (2013) Modeling human tumor angiogenesis in a three-dimensional culture system. Blood 121(21):e129-e137

41. Jain RK (2014) Antiangiogenesis strategies revisited: from starving tumors to alleviating hypoxia. Cancer Cell 26(5):605-622

42. Kloepper J et al (2016) Ang-2/VEGF bispecific antibody reprograms macrophages and resident microglia to anti-tumor phenotype and prolongs glioblastoma survival. Proc Natl Acad Sci USA 113(16):4476-4481

43. Holash J et al (1999) Vessel cooption, regression, and growth in tumors mediated by angiopoietins and VEGF. Science 284(5422):1994-1998

44. di Tomaso E et al (2011) Glioblastoma recurrence after cediranib therapy in patients: lack of "rebound" revascularization as mode of escape. Cancer Res 71(1):19-28

45. Waugh DJ, Wilson C (2008) The interleukin-8 pathway in cancer. Clin Cancer Res 14(21):6735-6741

46. McCoy MG et al (2019) Endothelial cells promote 3D invasion of GBM by IL-8-dependent induction of cancer stem cell properties. Sci Rep 9(1):9069

47. Infanger DW et al (2013) Glioblastoma stem cells are regulated by interleukin- 8 signaling in a tumoral perivascular niche. Cancer Res 73(23):7079-7089

48. Sharma I et al (2018) IL-8/CXCR48/2 signalling promotes tumor cell proliferation, invasion and vascular mimicry in glioblastoma. J Biomed Sci 25(1):62
49. Lindberg OR et al (2016) GBM heterogeneity as a function of variable epidermal growth factor receptor variant III activity. Oncotarget 7(48):79101-79116

50. Glatz JF, van der Vusse GJ (1996) Cellular fatty acid-binding proteins: their function and physiological significance. Prog Lipid Res 35(3):243-282

51. Hyvonen $\mathrm{M}$ et al (2014) Novel target for peptide-based imaging and treatment of brain tumors. Mol Cancer Ther 13(4):996-1007

52. Le Joncour V et al (2019) Vulnerability of invasive glioblastoma cells to lysosomal membrane destabilization. EMBO Mol Med 11(6)

53. Hetz C, Chevet E, Oakes SA (2015) Proteostasis control by the unfolded protein response. Nat Cell Biol 17(7):829-838

54. Jabouille A et al (2015) Glioblastoma invasion and cooption depend on IRE1alpha endoribonuclease activity. Oncotarget 6(28):24922-24934

55. Auf $\mathrm{G}$ et al (2010) Inositol-requiring enzyme 1alpha is a key regulator of angiogenesis and invasion in malignant glioma. Proc Natl Acad Sci USA 107(35):15553-15558

56. Hall A (1998) Rho GTPases and the actin cytoskeleton. Science 279(5350):509-514

57. Pasquale EB (2010) Eph receptors and ephrins in cancer: bidirectional signalling and beyond. Nat Rev Cancer 10(3):165-180

58. Krusche, B., et al., EphrinB2 drives perivascular invasion and proliferation of glioblastoma stem-like cells. Elife, 2016. 5

59. Torres VI, Godoy JA, Inestrosa NC (2019) Modulating Wnt signaling at the root: porcupine and Wnt acylation. Pharmacol Ther 198:34-45

60. Lu-Emerson $\mathrm{C}$ et al (2015) Lessons from anti-vascular endothelial growth factor and anti-vascular endothelial growth factor receptor trials in patients with glioblastoma. J Clin Oncol 33(10):1197-1213

61. Amoozgar Z, Jain RK, Duda DG (2019) Role of apelin in glioblastoma vascularization and invasion after anti-VEGF therapy: what is the impact on the immune system? Cancer Res 79(9):2104-2106

62. Martens $\mathrm{T}$ et al (2008) Inhibition of glioblastoma growth in a highly invasive nude mouse model can be achieved by targeting epidermal growth factor receptor but not vascular endothelial growth factor receptor-2. Clin Cancer Res 14(17):5447-5458

63. Munn LL, Jain RK (2019) Vascular regulation of antitumor immunity. Science 365(6453):544-545

64. Fukumura D et al (2018) Enhancing cancer immunotherapy using antiangiogenics: opportunities and challenges. Nat Rev Clin Oncol 15(5):325-340

Publisher's Note Springer Nature remains neutral with regard to jurisdictional claims in published maps and institutional affiliations. 